\title{
Automated multi-model deep neural network for sleep stage scoring with unfiltered clinical data
}

\author{
Xiaoqing Zhang ${ }^{1,2,3} \cdot$ Mingkai $\mathrm{Xu}^{4} \cdot$ Yanru $\mathrm{Li}^{1,2,3} \cdot$ Minmin Su $\mathrm{Su}^{1,2,3} \cdot$ Ziyao Xu ${ }^{4} \cdot$ Chunyan Wang ${ }^{1,2,3} \cdot$ Dan Kang ${ }^{1,2,3} \cdot$ \\ Hongguang $\mathrm{Li}^{1,2,3} \cdot \mathrm{Xin} \mathrm{Mu}^{1,2,3} \cdot$ Xiu Ding ${ }^{1,2,3} \cdot$ Wen $\mathrm{Xu}^{1,2,3} \cdot$ Xingjun Wang ${ }^{4} \cdot$ Demin Han ${ }^{1,2,3}$
}

Received: 15 October 2019 / Revised: 18 December 2019 / Accepted: 20 December 2019 / Published online: 14 January 2020

(C) The Author(s) 2020

\begin{abstract}
Purpose To develop an automated framework for sleep stage scoring from PSG via a deep neural network.

Methods An automated deep neural network was proposed by using a multi-model integration strategy with multiple signal channels as input. All of the data were collected from one single medical center from July 2017 to April 2019. Model performance was evaluated by overall classification accuracy, precision, recall, weighted F1 score, and Cohen's Kappa.

Results Two hundred ninety-four sleep studies were included in this study; 122 composed the training dataset, 20 composed the validation dataset, and 152 were used in the testing dataset. The network achieved human-level annotation performance with an average accuracy of 0.8181 , weighted F1 score of 0.8150 , and Cohen's Kappa of 0.7276 . Top- 2 accuracy (the proportion of test samples for which the true label is among the two most probable labels given by the model) was significantly improved compared to the overall classification accuracy, with the average being 0.9602 . The number of arousals affected the model's performance. Conclusion This research provides a robust and reliable model with the inter-rater agreement nearing that of human experts. Determining the most appropriate evaluation parameters for sleep staging is a direction for future research.
\end{abstract}

Keywords Polysomnography (PSG) · Obstructive sleep apnea (OSA) · Sleep staging · Deep learning

Xiaoqing Zhang and Mingkai Xu contributed equally to this work.

Electronic supplementary material The online version of this article (https://doi.org/10.1007/s11325-019-02008-w) contains supplementary material, which is available to authorized users.

Xingjun Wang

wangxingjun@tsinghua.edu.cn

Demin Han

deminhan_ent@hotmail.com

1 Beijing Tongren Hospital, Capital Medical University, Beijing 100730, People's Republic of China

2 Obstructive Sleep Apnea-Hypopnea Syndrome Clinical Diagnosis and Therapy and Research Centre, Capital Medical University, Beijing 100730, People's Republic of China

3 Key Laboratory of Otolaryngology Head and Neck Surgery, Ministry of Education, Capital Medical University, Beijing 100730, People's Republic of China

4 Department of Electronic Engineering, Tsinghua Shenzhen International Graduate School, Tsinghua University, Shenzhen, China

\section{Introduction}

Obstructive sleep apnea (OSA) is a disease characterized by recurrent partial or complete upper airway collapse obstruction during sleep, which can cause repeated apnea and hypopnea, often accompanied by hypoxemia, sleep disturbance, hypertension, coronary heart disease, and diabetes. OSA is the source of various cardiovascular and cerebrovascular diseases, endocrine diseases, and throat diseases. Epidemiological studies revealed that 936 million people worldwide suffer from moderate to severe OSA, and the number of people affected in China is among the highest in the world, causing a substantial social and economic burden [1]. Furthermore, studies suggest that $80 \%-90 \%$ of cases remain undiagnosed [1]. Therefore, it is crucial to improve the efficacy of diagnosis of OSA.

The diagnosis of OSA relies on overnight polysomnography (PSG) and manual data analysis in sleep laboratories. Sleep stage scoring criteria are standardized and follow the latest updates from the American Academy of Sleep Medicine (AASM) [2]. However, sleep stage scoring still relies on manual interpretation from skillful technicians. Thus, the traditional PSG 
scoring is time consuming [3], and therefore an automated sleep staging system would assist sleep experts and provide great clinical utility.

Deep learning, as a field in machine learning research, has undergone an expansion of its application space in recent years, promoting rapid analysis of complex image data; assisting in the screening, diagnosis, and follow-up of related diseases; and significantly shortening the diagnostic time with limited medical resources. Electroencephalography (EEG) is a nonstationary signal and has a low signal-to-noise ratio (SNR), but new ways are needed to improve EEG processing to achieve better generalization capabilities and more flexible application. Recently, deep learning (DL) has shown great promise in identifying EEG signals due to its capacity to learn good feature representations from raw data. The majority of studies tackling this issue adopt convolutional neural networks (CNNs), recurrent neural networks (RNNs), or a $\mathrm{CNN}+\mathrm{RNN}$ as the neural network architecture for sleep staging [4], and an accuracy rate greater than $87 \%$ has been reached [4].

In clinical settings, the scoring of sleep staging is complicated because the PSG processing could be confronted with challenging conditions, such as electrode shedding, signal artifacts, and noise. In this study, we use unfiltered clinical data and deep learning to develop automated analysis algorithms and validate them and to explore the scope of application in clinical practice.

\section{Materials and methods}

This retrospective study was approved by the institutional review board of Beijing Tongren Hospital (TRECKY2017032).

\section{Subjects}

All of the subjects were 18-70 years of age and had a history of habitual snoring. All of the subjects underwent overnight PSG in the sleep medicine center, Beijing Tongren Hospital from July 2017 to April 2019. Patient demographics were obtained for all of the subjects. The training dataset, validation dataset, and testing dataset were independent of one another based on inspection time. Patients less than 14 years old or had a time in bed (TIB) less than $4 \mathrm{~h}$ were excluded. Details are summarized in Table 1.

\section{Polysomnography}

Overnight, PSG was performed on all of the participants by the Philips Respironics G3 sleep diagnostic system, including a 2-channel electroencephalography (EEG) (C3/A2, C4/A1), 2-channel electrooculography (EOG), anterior tibial electromyogram (EMG), electrocardiogram (ECG), 2channel airflow measurement with nasal cannula pressure, recording of respiratory (thoracic and abdominal) movements, and pulse oximetry for oxygen saturation $\left(\mathrm{SpO}_{2}\right)$. All of the ECG and EOG channels were captured at a $200 \mathrm{~Hz}$ sampling frequency and displayed with a $0.3-35 \mathrm{~Hz}$ band-pass filter. Anterior tibial EMG had a sampling rate of $200 \mathrm{~Hz}$, and the band-pass filter was $10-100 \mathrm{~Hz}$.

Two highly trained, experienced (more than 10 years) PSG technologists scored sleep stages and respiratory events in $30 \mathrm{~s}$ epoch in accordance with the American Association of Sleep Medicine (AASM 2012) guidelines [5]. The apnea-hypopnea index (AHI) was defined as the number of apnea and hypopnea events per hour of sleep and was used to indicate the severity of sleep apnea (normal: $\mathrm{AHI}<5$; mild OSA, $5 \leq$ $\mathrm{AHI}<15$; moderate OSA, $15 \leq \mathrm{AHI}<30$; severe OSA, $\mathrm{AHI} \geq 30$ ).

\section{Data processing}

According to the AASM standard, the central band of the EEG signal is concentrated below $35 \mathrm{~Hz}$, while the sampling rate is $200 \mathrm{~Hz}$. Instead of getting more information from the excessive sampling frequency, we only get high-frequency noise. Therefore, we first filtered the signal at $66 \mathrm{~Hz}$ and then downsampled the signal sampling frequency to $66 \mathrm{~Hz}$ (which is one-third of the original sampling frequency) to remove the influence of high-frequency noise while ensuring that no spectral aliasing occurs and to reduce the amount of data.

Considering the sleep continuity, the staging of each epoch may correlate with the previous and subsequent epochs. A 90$\mathrm{s}$ window (3 epochs) to redivide the signal was applied with a stride of $30 \mathrm{~s}$, which means that newly divided epoch's length was three times the original length. The newly divided epoch took the stage label of the original $30 \mathrm{~s}$ epoch as its label (Fig. $1)$.

\section{Neural network}

(The details are in the supplementary materials)

\section{Training}

(The details are in the supplementary materials)

\section{Noise detection}

(The details are in the supplementary materials)

\section{Expert rules}

The REM stage is exceptional in EEG staging. Although the REM stage has specific characteristics, rapid eye movements 
Table 1 Demographics and characteristics of datasets

\begin{tabular}{|c|c|c|c|c|}
\hline & Training & Validation & Testing & $P$ value \\
\hline Number of participants/epochs & $92 / 93,788$ & $21 / 20,845$ & $152 / 150103$ & \\
\hline Normal & $13 / 13081$ & $3 / 2958$ & $23 / 22741$ & \\
\hline Mild OSA & 19/19612 & $4 / 3976$ & $23 / 22580$ & \\
\hline Moderate OSA & $17 / 17163$ & $4 / 4262$ & $29 / 27774$ & \\
\hline Severe OSA & $43 / 43932$ & $10 / 9649$ & $77 / 77008$ & \\
\hline Sex (male: female) & $64: 28$ & $16: 4$ & $129: 33$ & $>0.05$ \\
\hline Age (median, range) & $42.5(19-68)$ & $47.5(22-57)$ & $38.0(79-61)$ & $<0.05^{*}$ \\
\hline BMI $\left(\mathrm{kg} / \mathrm{m}^{2}\right)$ (median, range) & $25.95(16.1-38.4)$ & $27.65(18.8-34.0)$ & $26.55(13.8-46.3)$ & $>0.05$ \\
\hline TST (min) (median, range) & $423.10(200.5-577.6)$ & $436.10(285.5-510.4)$ & $426.85(92.0-578.5)$ & $>0.05$ \\
\hline \multicolumn{5}{|l|}{ AHI (median, range) } \\
\hline Normal & $1.8(0.5-4.2)$ & $1.2(0.6-2.2)$ & $1.4(0.2-4.9)$ & $>0.05$ \\
\hline Mild OSA & $11.1(5.4-13.5)$ & $9.7(7.7-14.3)$ & $9.1(5.4-14.1)$ & $>0.05$ \\
\hline Moderate OSA & $19.9(15.3-29.2)$ & $18.45(15.1-29.7)$ & $23.7(15.1-28.8)$ & $>0.05$ \\
\hline Severe OSA & $51.8(30.6-105.3)$ & $66.6(37.3-97.7)$ & $56.9(30.9-112.4)$ & $>0.05$ \\
\hline \multicolumn{5}{|l|}{ Sleep stage $(n, \%)$} \\
\hline $\mathrm{W}$ & 16,201 & 2339 & 26,112 & \\
\hline N1 & 14,839 & 3574 & 24,489 & \\
\hline $\mathrm{N} 2$ & 47,889 & 10,744 & 73,395 & \\
\hline N3 & 1881 & 648 & 3289 & \\
\hline $\mathrm{R}$ & 12,978 & 3540 & 22,818 & \\
\hline $\begin{array}{l}\text { Minimum } \mathrm{SpO}_{2}(\%) \\
\text { (median, range) }\end{array}$ & $85(51-96)$ & 83 (37-94) & $83(35-95)$ & $>0.05$ \\
\hline $\begin{array}{l}\text { Number of arousals } \\
\text { (median, range) }\end{array}$ & $79.5(1-592)$ & $97(7-528)$ & $79.5(0-692)$ & $>0.05$ \\
\hline
\end{tabular}

$B M I$ body mass index, $T S T$ total sleep time, $A H I$ apnea-hypopnea index, $\mathrm{SpO}_{2}$ pulse oxygen saturation

do not occur within every 30 -s epochs. However, it is quite difficult for the model to determine whether these epochs with no rapid eye movements are in the REM stage because it relies on prior knowledge of the current stage. Therefore, we checked each epoch's next eight epochs: if there was a REM stage epoch, we forcibly converted this epoch to the REM stage, thus ensuring the continuity of the REM period.

\section{Model architecture}

The overall algorithm framework is shown in Fig. 2. After preprocessing, the signal was input into the corresponding CNN model and the real-time noise detection module. The outputs of multiple models were integrated while setting the weights of the falloff signal models to zero. Then the model modified the integrated prediction of multiple models by expert-defined rules to get the final prediction.

\section{Model evaluation and statistical analysis}

The performance of sleep stage prediction was measured by overall classification accuracy, precision, recall, weighted F1 score, and Cohen's Kappa. Top-2 accuracy was applied, which means that the two most probable predictions for the model prediction were considered "correct."

The confusion matrix was applied to the visualization of the performance of algorithms.

Statistical analysis was performed using SPSS 25 software (SPSS Inc., Chicago, IL). The Shapiro-Wilk test was used to verify normal value distribution. Differences in variables were analyzed by Student's t-test or Mann-Whitney U test. All of the $P$ values were 2 -sided, and $P$ values less than 0.05 were considered to be significant.

\section{Cross dataset experiments}

To further evaluate the performance of our method, we evaluated it on a public dataset named Sleep-EDF. In order to compare our method with others, we used the 2013 version, which contains two sets of subjects from two studies: age effect in healthy subjects (SC) and Temazepam effects on sleep (ST). Two PSGs of about $20 \mathrm{~h}$ each were recorded during two subsequent daynight periods at the subjects' homes. Well-trained technicians manually scored corresponding hypnograms (sleep patterns) according to the Rechtschaffen and Kales 


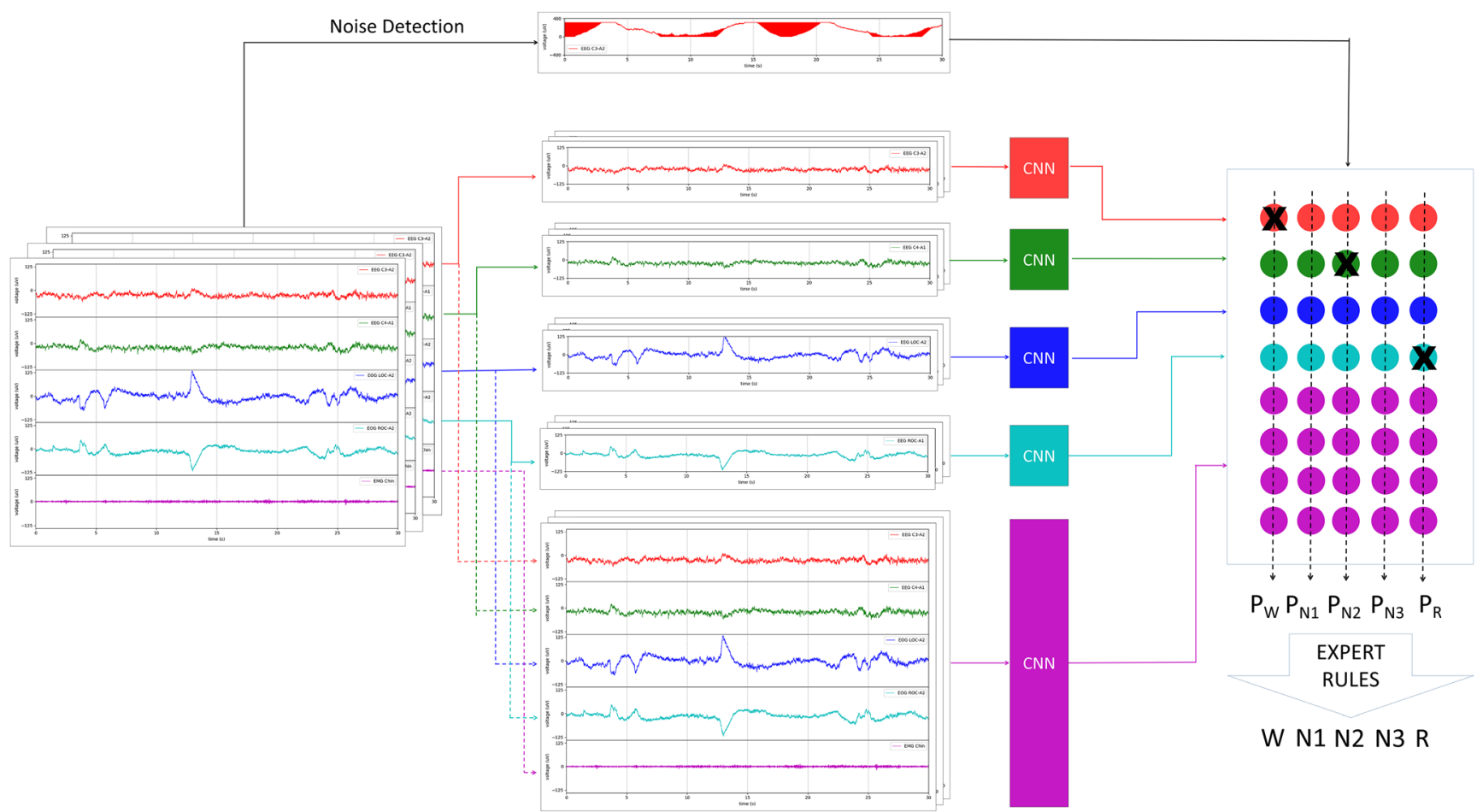

Fig. 1 Overall architecture of our method. The left side shows the input signals, consisting of 5 channels: EEG C3/A2, EEG C4/A1, 2-channel $\mathrm{EOG}$, and EMG. The 5-channel signal is divided into 5 groups, as shown in the middle of the figure: Groups 1 to 4 are EEG C3/A2, EEG C4/A1, and 2-channel EOG, respectively, and the fifth group consists of all 5 input signals. Then each group of signals was feed into a CNN model for training and prediction. At the same time, a noise detection algorithm detected the noise in each group. The right part of the illustration shows the integration, and the colored nodes represent integration weights corresponding to different $\mathrm{CNN}$ models. We take the weighted-average as each stage's probability. Notice that the " $\mathrm{X}$ " on the weight means that this weight is reset to zero due to noise. After integration, the output prediction was modified by expert-defined rules manual. As AASM recommends, N3 and N4 of the sleep-EDF dataset were merged in this study. Twenty in-bed SC subjects (age 28.7 \pm 2.9 ) were used. Each PSG recording contained 2 scalp-EEG signals (Fpz-Cz and $\mathrm{Pz}-\mathrm{Cz}$ ), 1 EOG (horizontal), 1 EMG, and 1 oralnasal respiration signal. All EEG and EOG had the same sampling rate of $100 \mathrm{~Hz}$. The SC dataset was divided into five folds for training and independent validation.

\section{Result}

\section{Population characteristics}

The numbers of PSG subjects in the training dataset, the validation dataset, and the testing dataset were 122, 20, and 152 , respectively. Of the three datasets, males accounted for the vast majority. No significant differences were detected in sex, BMI, total sleep time, AHI, sleep stage distribution, minimum $\mathrm{SpO} 2$, and number of arousals, suggesting that the samples in the three datasets were homogeneous. The only significant difference was detected in age.

\section{Comparative study to choose the best algorithm}

To select the most appropriate model, comparative studies were conducted to evaluate the same testing dataset; the results are summarized in Table 2. Models with neither the 3epoch splice, expert rules nor noise detection resulted in lower evaluation parameters (Fig. 2).

\section{Model performance}

Table 3 presents more detailed results of the model described above. The average predicted TST was $410.18 \mathrm{~min}$, compared $426.85 \mathrm{~min}$ calculated by human experts. The population of the testing dataset was divided into four groups according to the degree of AHI. The normal population received the highest accuracy and the highest weighed F1 score. The confusion matrix demonstrated that the most appropriate model after comparative studies possesses higher consistency for W, N2, and $\mathrm{R}$ identification but has poor performance for $\mathrm{N} 1$ and N3 (Fig. 3). Moreover, the F1 score and Cohen's Kappa indicated moderate to strong inter-rater agreement between the model performance and human experts on 


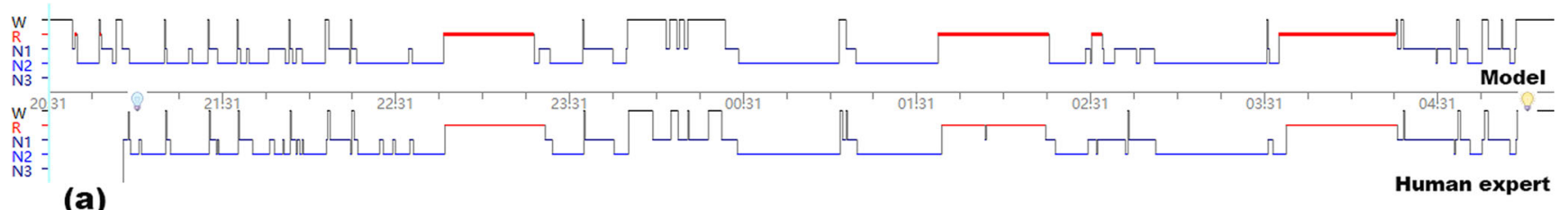

(a)

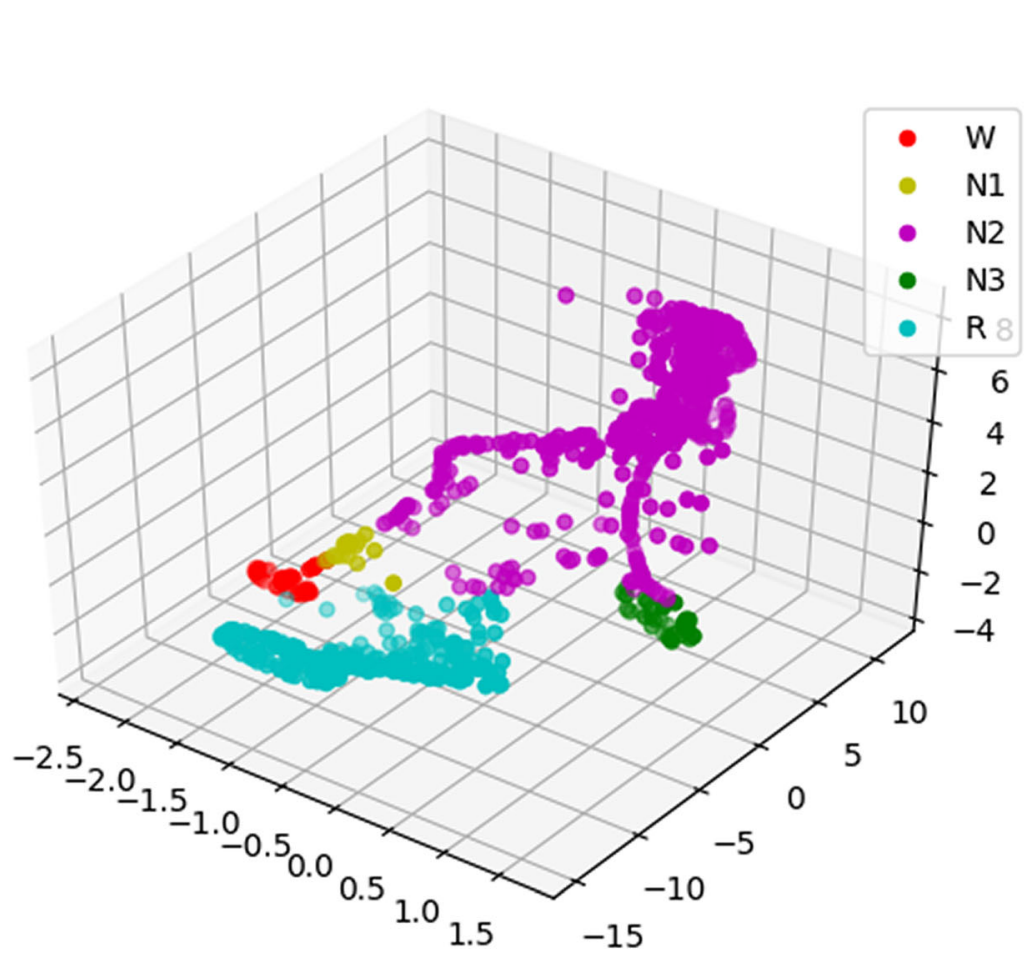

(b)

Fig. 2 (a) Example of an overnight PSG record scored by the model vs. human expert. (b) t-SNE for the last hidden layer of the CNN. Each differently colored point indicates a sleep stage scored by the model, suggesting that the model can discriminate different sleep stages well

weighted average performance for both classification by AHI and by sleep stage (Table 4).

\section{Top-2 accuracy on sleep stage scoring}

Since there is inter-rater variability between technicians in sleep staging, in this study, we introduce an evaluation index called the top- 2 accuracy, defined as the proportion of test samples for which the correct label is among the two most probable labels given by the model. The neural network is an appropriate method mathematically; in classification tasks, it judges the similarity between the input sample and the data distribution corresponding to each label, scores the similarities, and normalizes them into probability. If the correct label of a sample is among the two most probable labels given by the neural network, we consider this sample as an exact sample in the context of the top-2 accuracy (Table 5).

Table 2 Model performance with different training algorithms

\begin{tabular}{llll}
\hline Training algorithm & Macro-accuracy & Weighted F1 score & Cohen's Kappa \\
\hline Without the 3-epoch splice & 0.8034 & 0.7885 & 0.7044 \\
Without noise detection & 0.8050 & 0.7996 & 0.7105 \\
Without expert rules & 0.8173 & 0.8115 & 0.7266 \\
The proposed model & 0.8181 & 0.8150 & 0.7276 \\
\hline
\end{tabular}

AHI apnea-hypopnea index 


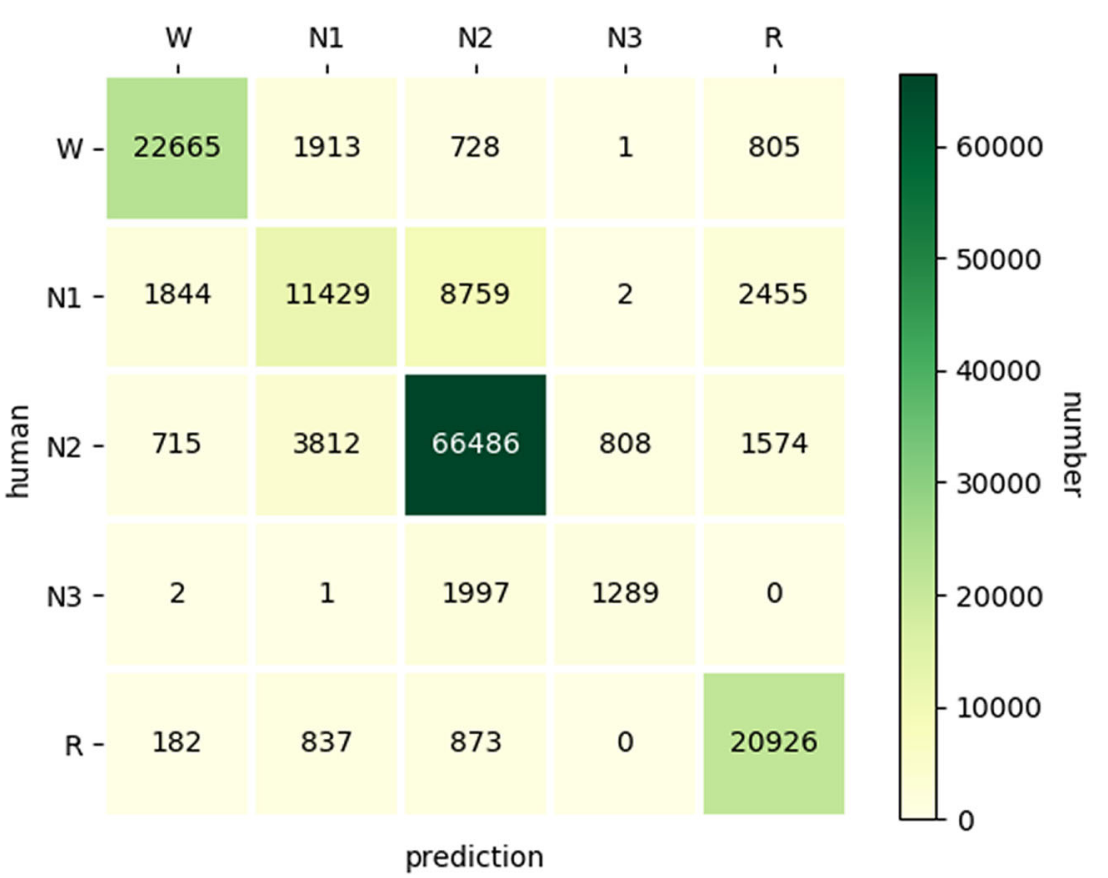

Fig. 3 Confusion matrix for the predicted sleep stage, displaying the agreement with expert scores. The vertical rows represent the sleep staging scored by the human expert, while the horizontal rows are the predictions for the same epoch of the testing dataset. The diagonal

numbers are the epochs for which the prediction of the model matches the human expert at each sleep stage. The model possesses higher consistency for $\mathrm{W}, \mathrm{N} 2$, and R identification

Table 6 presents the model's predictions for each epoch with the two most probable predictions. For example, the difference between N1 and W in EEG is sometimes not obvious, and different technicians will have different judgments. The model in this paper has a judgment ability close to that of a human technician for the confusing EEG. The most striking result was that when the model output $\mathrm{N} 2$, the second possible sleep stage was N1 stages accounted for $66.79 \%$. Similarly, the second possible sleep stage was N2 when the model outputs N3 in most instances. Since the REM stages can be divided into the phasic mode and tonic mode, the second identification of the model distributed in the $\mathrm{W}$ stages, N1 stages, and N2 stages, accounts for the majority.
In order to explore the reasons behind this, we try to divide the data in the testing dataset into two groups: the increased rate of accuracy greater or equal to the average increase rate $(0.1419)$ and the increase rate smaller than the average. Statistical analysis showed that the greater the number of arousals, the higher the top-2 accuracy. In other words, the number of arousals affected the model's performance on sleep staging (Fig. 4).

\section{Evaluation of cross dataset experiments}

Studies comparing the proposed model to other methods on sleep-EDF are summarized in Table 7. The model showed improvement in all metrics. Compared with the testing dataset results from our clinical center, the per-class F1 of the N3

Table 3 Model performance on testing dataset according to AHI

\begin{tabular}{llll}
\hline Testing dataset & Macro-accuracy & Weighted-F1 score & Cohen's Kappa \\
\hline Normal & 0.8361 & 0.8277 & 0.7560 \\
Mild OSA & 0.8265 & 0.8221 & 0.7433 \\
Moderate OSA & 0.8222 & 0.8153 & 0.7288 \\
Severe OSA & 0.8088 & 0.7981 & 0.7124 \\
Weighted average & 0.8181 & 0.8150 & 0.7276 \\
\hline
\end{tabular}

AHI apnea-hypopnea index 
Table 4 Model performance on sleep staging of testing dataset

\begin{tabular}{ccccc}
\hline Sleep staging & Precision & Recall & F1 score & Number of epochs \\
\hline W & 0.8920 & 0.8680 & 0.8799 & 25,408 \\
N1 & 0.6352 & 0.4667 & 0.5381 & 17,992 \\
N2 & 0.8433 & 0.9059 & 0.8734 & 78,843 \\
N3 & 0.6138 & 0.3919 & 0.4784 & 2100 \\
R & 0.8123 & 0.9171 & 0.8615 & 25,760 \\
Weighted average & 0.8181 & 0.8150 & 0.7276 & 150,103 \\
\hline
\end{tabular}

stages was significantly improved. However, the per-class F1 of the N1 period was still lower than that of other sleep stages, which is consistent with the findings of other studies [6-8].

\section{Discussion}

In this study, the model performed robustly under different levels of AHI and performed slightly better in the healthy population than in patients with severe OSA. As the AHI increased, the accuracy and F1 values gradually decreased. In patients with severe OSA, the lowest value is considered to be related to fragmented sleep, and the EEG is relatively complicated. Cohen's Kappa was to evaluate the inter-rater variability between the model and the technician's scoring. The literature suggests that there is inter-rater variability between different human technicians, and both $\mathrm{N} 1$ and $\mathrm{N} 3$ are relatively low, ranging from $20 \%$ to $70 \%$ [9-12]. The average Cohen's Kappa of this study was 0.7276 , indicating a substantial agreement with human technicians. Similar to the previous pieces of literature, the model displayed a low consistency in the N1 and N3 stages. Such a result considers that the waveform characteristics of the low amplitude in the N1 stage are not prominent, and the model may confuse N1 with N2 during scoring (like when the EEG is not typical and thus a technician confuses $\mathrm{N} 1$ and N2). However, the agreement of N3 is weak due to the high proportion of OSA patients in the training dataset, which may lead to the number of N3 periods being inadequate, accounting for only $2 \%$ of the total number of epochs. In clinical practice, the number of sleep stages in clinical data is imbalanced. Compared with healthy people, sleep fragmentation in OSA patients has more $\mathrm{W}$ and N1 stages and fewer $\mathrm{N} 3$ stages. In this study, because the unfiltered data was closer to the clinical situation, the imbalanced sample categories will result in too few features and too diminutive a sample size to extract the data pattern, or in over-fitting problems because of limited samples. For the test of the public dataset, the metrics were significantly improved in N3 stages

To determine the final model architecture, this study conducted a comparative study on the same testing dataset. In the clinical PSG, there may be a decrease in signal quality due to sweating, intolerance to the environment, limb movement, and so forth. Therefore, the model design of this study considers the possibility of abnormal signal acquisition during overnight sleep PSG. Second, since there are transitional rules associated with the sleep staging, Markov models, CNNs, and RNNs have been used in recognition of sleep EEG in recent years [13-17]. This research innovatively applied the method of three-epoch splicing to simulate the technician recognition of EEG, so that if there is an epoch with atypical or severe interference, technicians could refer to the previous and following epochs of the EEG. Another innovation in this study is the addition of expert rules. In clinical practice, the identification of REM stages mainly includes rapid eye movement, low-tension diaphragmatic electromyography, sawtooth waves, and transient myoelectric activity. The tonic mode of REM sleep should not have any apparent ocular activity so that the model does not make erroneous judgments. Expert rules can substantially avoid erroneous judgments.

To explore the analysis process of the model, this study innovatively introduced the concept of top- 2 accuracy. As a result, the overall accuracy was dramatically improved. Through the analysis of the predicted value of the second probability of the model, this study finds that the model will have a certain degree of confusion when distinguishing between the $\mathrm{W}$ and $\mathrm{N} 1$ stages, between the $\mathrm{N} 1$ and $\mathrm{N} 2$ stages, and between N2

Table 5 Model performance on testing dataset

\begin{tabular}{llll}
\hline Testing dataset & Top-1 macro-accuracy & Top-2 macro-accuracy & Average increase rate \\
\hline Normal & 0.8341 & 0.9611 & 0.1270 \\
Mild & 0.8292 & 0.9698 & 0.1407 \\
Moderate & 0.8228 & 0.9512 & 0.1285 \\
Severe & 0.8088 & 0.9619 & 0.1531 \\
The average performance & 0.8184 & 0.9602 & 0.1419 \\
\hline
\end{tabular}

AHI apnea-hypopnea index 
Fig. 4 According to the growth rate of accuracy, there was no statistical difference in AHI between the two groups, but the number of arousals demonstrated significant differences $(\mathrm{p}<0.01)$. AHI=Apnea-Hypopnea Index

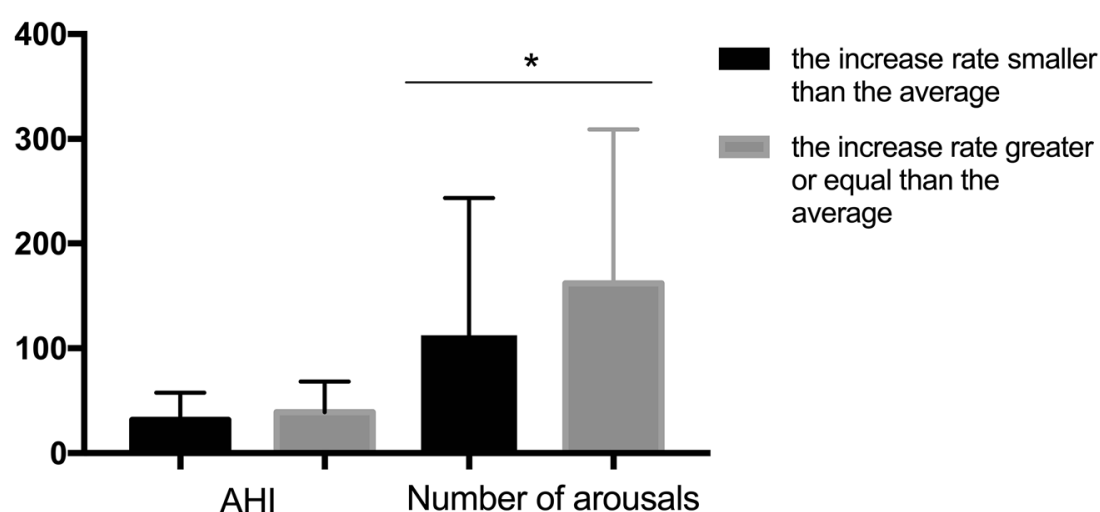

and N3 stages; this is consistent with the most common differences in sleep scoring by human experts [18]. A previous study pointed out that the definition of divergence and the K complex wave lacks specificity and is related to the existence of spindle wave identification [19]. Since the lack of a clear "absolute truth value" for sleep stage scoring, the substantial increase in top-2 accuracy indicates that the model output is reasonable. This study found that arousal affects the accuracy of sleep staging; this may be due to the number of arousals being positively correlated with the number of N1 stages [20]. Moreover, the model performance of $\mathrm{N} 1$ is lower than that of other sleep stages. This study proposes a future direction for the evaluation of deep learning algorithms by analyzing the top two rankings of maximum probability values for sleep staging. For sleep staging, which relies on manual scoring and must consider inter-rater variability, it is worthwhile to study which parameters are chosen to evaluate model performance. Three classifications (awakening, NREM, and REM) or four classifications (awakening, shallow sleep $(\mathrm{N} 1+\mathrm{N} 2)$, deep sleep (N3), and REM)) make sense in clinical practice.

There are some limitations to this study. First, the clinical data of this study is imbalanced, and the number of N3 stages in this study is small. Compared with other studies, the recognition of N3 is lower. Second, the clinical dataset used in this study was derived from retrospective data of a single center, lacking analysis of homogeneity with the published dataset sleep-EDF. Additionally, the study applied independent and homogeneous training sets and testing sets without cross-validation, and thus there may be deficiencies in the assessment of the generalization capabilities of the model.

\section{Conclusion}

In conclusion, this research provides a robust and reliable model in which the inter-rater agreement nears that of human experts. In future research, it is essential to address the abovementioned limitations, explore the evaluation criteria for neural network models, and develop a lightweight version of the model to make it work in wearable devices and smart devices. Eventually, this work can have a positive impact on population health and healthcare expenditures.

Acknowledgments This research was supported by the National Key Research \& Development Program of China (2017YFC0112500), Beijing Municipal Administration of Hospitals' Mission Plan (SML20150201), and Beijing Municipal Administration of Hospitals Incubating Program (PX 2019005). Xiaoqing Zhang and Mingkai Xu contributed equally to this work. The first draft of the manuscript was written by Xiaoqing Zhang and Mingkai Xu. Demin Han and Xingjun Wang are co-corresponding authors of this paper.

Table 6 Distribution of sleep staging with two most significant predicted probabilities for each epoch (without expert rules)

\begin{tabular}{|c|c|c|c|c|c|c|}
\hline \multirow[t]{2}{*}{ Number of epochs $(\% *)$} & & \multicolumn{5}{|c|}{ The second largest probability of prediction } \\
\hline & & $\mathrm{W}$ & $\mathrm{N} 1$ & $\mathrm{~N} 2$ & N3 & $\mathrm{R}$ \\
\hline \multirow[t]{5}{*}{ Maximum probability of prediction (model output) } & $\mathrm{W}$ & - & $11,984(47.17 \%)$ & $629(2.47 \%)$ & $15(0.06 \%)$ & $2119(8.33 \%)$ \\
\hline & $\mathrm{N} 1$ & $3994(22.20 \%)$ & - & $6294(34.98 \%)$ & $0(0 \%)$ & $2795(15.53 \%)$ \\
\hline & $\mathrm{N} 2$ & $1030(13.06 \%)$ & $52,656(66.79 \%)$ & - & $11,347(14.39 \%)$ & $1392\left(9.1 \times 10^{-4} \%\right)$ \\
\hline & $\mathrm{N} 3$ & $2(0.09 \%)$ & 0 & $1289(61.38 \%)$ & - & 0 \\
\hline & $\mathrm{R}$ & $1265(6.91 \%)$ & $14,886(57.79 \%)$ & $3577(13.89 \%)$ & $0(0 \%)$ & - \\
\hline
\end{tabular}

*Proportion of the same epoch of the testing dataset 
Table 7 Comparation of other methods to the proposed method

\begin{tabular}{|c|c|c|c|c|c|c|c|c|c|}
\hline \multirow[t]{2}{*}{ Method } & \multirow[t]{2}{*}{ Channel } & \multirow[t]{2}{*}{ Acc } & \multirow[t]{2}{*}{ Macro-average F1 } & \multicolumn{5}{|c|}{ Per-class F1 } & \multirow{2}{*}{$\begin{array}{l}\text { Cohen's } \\
\text { Kappa }\end{array}$} \\
\hline & & & & W & N1 & $\mathrm{N} 2$ & N3 & $\mathrm{R}$ & \\
\hline Supratak A et al. [7] & $\mathrm{Fpz}-\mathrm{Cz}$ & 0.820 & 0.769 & 0.847 & 0.466 & 0.859 & 0.848 & 0.824 & 0.76 \\
\hline Supratak A et al. [7] & $\mathrm{Pz}-\mathrm{Oz}$ & 0.798 & 0.731 & 0.881 & 0.370 & 0.827 & 0.773 & 0.803 & 0.72 \\
\hline Tsinalis O et al. [6] & $\mathrm{Fpz}-\mathrm{Cz}$ & 0.789 & 0.737 & 0.716 & 0.370 & 0.846 & 0.840 & 0.814 & 0.65 \\
\hline Sun Y, et al. [8] & $\mathrm{Pz}-\mathrm{Oz}$ & 0.810 & 0.736 & 0.856 & 0.249 & 0.889 & 0.792 & 0.863 & 0.73 \\
\hline The proposed model & All & 0.836 & 0.781 & 0.864 & 0.498 & 0.887 & 0.845 & 0.816 & 0.77 \\
\hline
\end{tabular}

Acc accuracy

Authors' contributions The first draft of the manuscript was written by Xiaoqing Zhang and Mingkai Xu. All authors commented on previous versions of the manuscript. All authors read and approved the final manuscript.

Funding information This research was supported by the National Key Research \& Development Program of China (2017YFC0112500), Beijing Municipal Administration of Hospitals' Mission Plan (SML20150201), and Beijing Municipal Administration of Hospitals Incubating Program (PX 2019005).

\section{Compliance with ethical standards}

Conflict of interest The authors declare that they have no conflict of interest.

Ethical approval All procedures performed in studies involving human participants were in accordance with the ethical standards of the institutional and/or national research committee and with the 1964 Helsinki declaration and its later amendments or comparable ethical standards.

Informed consent Informed consent was obtained from all individual participants' guardians included in the study.

Open Access This article is licensed under a Creative Commons Attribution 4.0 International License, which permits use, sharing, adaptation, distribution and reproduction in any medium or format, as long as you give appropriate credit to the original author(s) and the source, provide a link to the Creative Commons licence, and indicate if changes were made. The images or other third party material in this article are included in the article's Creative Commons licence, unless indicated otherwise in a credit line to the material. If material is not included in the article's Creative Commons licence and your intended use is not permitted by statutory regulation or exceeds the permitted use, you will need to obtain permission directly from the copyright holder. To view a copy of this licence, visit http://creativecommons.org/licenses/by/4.0/.

\section{References}

1. Benjafield AV, Ayas NT, Eastwood PR, Heinzer R, Ip MSM, Morrell MJ, Nunez CM, Patel SR, Penzel T, Pepin JL, Peppard PE, Sinha S, Tufik S, Valentine K, Malhotra A (2019) Estimation of the global prevalence and burden of obstructive sleep apnoea: a literature-based analysis. Lancet Respir Med 7(8):687-698. https:// doi.org/10.1016/S2213-2600(19)30198-5

2. Berry RB, Brooks R, Gamaldo C, Harding SM, Lloyd RM, Quan SF, Troester MT, Vaughn BV (2017) AASM scoring manual updates for 2017 (version 2.4). J Clin Sleep Med 13(5):665-666. https://doi.org/10.5664/jcsm.6576

3. Knauert M, Naik S, Gillespie MB, Kryger M (2015) Clinical consequences and economic costs of untreated obstructive sleep apnea syndrome. World J Otorhinolaryngol Head Neck Surg 1(1):17-27. https://doi.org/10.1016/j.wjorl.2015.08.001

4. Roy Y, Banville H, Albuquerque I, Gramfort A, Falk TH, Faubert J (2019) Deep learning-based electroencephalography analysis: a systematic review. J Neural Eng. https://doi.org/10.1088/17412552/ab260c

5. Berry RB, Budhiraja R, Gottlieb DJ, Gozal D, Iber C, Kapur VK, Marcus CL, Mehra R, Parthasarathy S, Quan SF, Redline S, Strohl KP, Davidson Ward SL, Tangredi MM, American Academy of Sleep M (2012) Rules for scoring respiratory events in sleep: update of the 2007 AASM manual for the scoring of sleep and associated events. Deliberations of the sleep apnea definitions task force of the American Academy of sleep medicine. J Clin Sleep Med 8(5):597619. https://doi.org/10.5664/jcsm. 2172

6. Tsinalis O, Matthews PM, Guo Y, Zafeiriou S (2016) Automatic sleep stage scoring with single-channel EEG using convolutional neural networks. arXiv preprint arXiv:161001683

7. Supratak A, Dong H, Wu C, Guo Y (2017) DeepSleepNet: a model for automatic sleep stage scoring based on raw single-channel EEG. IEEE Trans Neural Syst Rehabil Eng 25(11):1998-2008

8. Sun Y, Wang B, Jin J, Wang X (2018) Deep convolutional network method for automatic sleep stage classification based on neurophysiological signals. In: 2018 11th International Congress on Image and Signal Processing, BioMedical Engineering and Informatics (CISP-BMEI). IEEE, pp 1-5

9. Whitney CW, Gottlieb DJ, Redline S, Norman RG, Dodge RR, Shahar E, Surovec S, Nieto FJ (1998) Reliability of scoring respiratory disturbance indices and sleep staging. Sleep 21(7):749-757. https://doi.org/10.1093/sleep/21.7.749

10. Norman RG, Pal I, Stewart C, Walsleben JA, Rapoport DM (2000) Interobserver agreement among sleep scorers from different centers in a large dataset. Sleep 23(7):901-908

11. Collop NA (2002) Scoring variability between polysomnography technologists in different sleep laboratories. Sleep Med 3(1):43-47

12. Younes M, Kuna ST, Pack AI, Walsh JK, Kushida CA, Staley B, Pien GW (2018) Reliability of the American Academy of sleep medicine rules for assessing sleep depth in clinical practice. J Clin Sleep Med 14(2):205-213. https://doi.org/10.5664/jcsm.6934

13. Patanaik A, Ong JL, Gooley JJ, Ancoli-Israel S, Chee MWL (2018) An end-to-end framework for real-time automatic sleep stage classification. Sleep 41(5). https://doi.org/10.1093/sleep/zsy041 
14. Kang DY, DeYoung PN, Malhotra A, Owens RL, Coleman TP (2018) A state space and density estimation framework for sleep staging in obstructive sleep apnea. IEEE Trans Biomed Eng 65(6): 1201-1212. https://doi.org/10.1109/TBME.2017.2702123

15. Zhang L, Fabbri D, Upender R, Kent D (2019) Automated sleep stage scoring of the sleep heart health study using deep neural networks. Sleep. https://doi.org/10.1093/sleep/zsz159

16. Allocca G, Ma S, Martelli D, Cerri M, Del Vecchio F, Bastianini S, Zoccoli G, Amici R, Morairty SR, Aulsebrook AE, Blackburn S, Lesku JA, Rattenborg NC, Vyssotski AL, Wams E, Porcheret K, Wulff K, Foster R, Chan JKM, Nicholas CL, Freestone DR, Johnston LA, Gundlach AL (2019) Validation of 'Somnivore', a machine learning algorithm for automated scoring and analysis of polysomnography data. Front Neurosci 13:207. https://doi.org/10. 3389/fnins.2019.00207

17. Biswal S, Sun H, Goparaju B, Westover MB, Sun J, Bianchi MT (2018) Expert-level sleep scoring with deep neural networks. J Am
Med Inform Assoc 25(12):1643-1650. https://doi.org/10.1093/ jamia/ocy 131

18. Younes M, Raneri J, Hanly P (2016) Staging sleep in polysomnograms: analysis of inter-scorer variability. J Clin Sleep Med 12(6):885-894. https://doi.org/10.5664/jcsm.5894

19. Warby SC, Wendt SL, Welinder P, Munk EG, Carrillo O, Sorensen HB, Jennum P, Peppard PE, Perona P, Mignot E (2014) Sleepspindle detection: crowdsourcing and evaluating performance of experts, non-experts and automated methods. Nat Methods 11(4): 385-392. https://doi.org/10.1038/nmeth.2855

20. Gugger M, Molloy J, Gould GA, Whyte KF, Raab GM, Shapiro CM, Douglas NJ (1989) Ventilatory and arousal responses to added inspiratory resistance during sleep. Am Rev Respir Dis 140(5): 1301-1307. https://doi.org/10.1164/ajrccm/140.5.1301

Publisher's note Springer Nature remains neutral with regard to jurisdictional claims in published maps and institutional affiliations. 